CONFORMAL GEOMETRY AND DYNAMICS

An Electronic Journal of the American Mathematical Society

Volume 8, Pages 36-51 (March 8, 2004)

S $1088-4173(04) 00107-9$

\title{
THE INFORMATION TOPOLOGY AND TRUE LAMINATIONS FOR DIFFEOMORPHISMS
}

\author{
MEIYU SU
}

\begin{abstract}
We explore the lamination structure from data supplied by a general measure space $X$ provided with a Borel probability measure $m$. We show that if the data satisfy some typical axioms, then there exists a lamination $\mathcal{L}$ injected in the underlying space $X$ whose image fills up the measure $m$. For an arbitrary $C^{1+\alpha}$-diffeomorphism $f$ of a compact Riemannian manifold $M$, we construct the data that naturally possess the properties of the axioms; thus we obtain the stable and unstable laminations $\mathcal{L}^{s / u}$ continuously injected in the stable and unstable partitions $\mathcal{W}^{s / u}$. These laminations intersect at almost every regular point for the measure.
\end{abstract}

\section{INTRODUCTION}

In this paper, we study the lamination structure from data supplied by a general discrete time dynamical system. A lamination or laminated space is a topological space covered by "open sets" (called boxes) of the form $T \times$ (open $k$-disk) so that the overlap maps preserve the second factor. The lamination we construct will be invariant under the dynamics.

The data supplied by any diffeomorphism $f$ of a compact Riemannian manifold $M$ with positive entropy will be:

(i) a standard Borel space $X$ (the manifold or rather an appropriate subset $L \subset M)$

(ii) a measure $m$ on the Borel space $X$ (an $f$-invariant and ergodic measure $\mu$ of positive entropy);

(iii) a sequence of countable partitions $\mathcal{P}_{1} \prec \mathcal{P}_{2} \prec \ldots \prec \mathcal{P}_{n} \prec \ldots$ generating the Borel structure (the countable partition $\mathcal{P}$ of finite entropy constructed in [4]);

(iv) a countable family of boxes of the form $(T \times k$-disk $)$ which will cover $X$ (these will be generated by the Pesin boxes covering the regular set $\Re$ by applying the dynamics).

These data are subject to the Axioms described in $\S 2$, which are fulfilled in the quite interesting dynamical example because of 11 and 4 (see §3). Such axioms allow us to define a new topology on the transversals of the boxes (the information topology). To do this, we need to refine the boxes covering the support of the measure $m(\mu)$ by removing sets of horizontal disks of measure zero and then

Received by the editors September 10, 2003 and, in revised form, January 29, 2004.

2000 Mathematics Subject Classification. Primary 37D30; Secondary 37C05.

Key words and phrases. $C^{1+\alpha}$-diffeomorphisms on Riemannian manifolds, stable and unstable manifolds and partitions, laminations, Pesin boxes, and information topology.

(C)2004 American Mathematical Society 
saturating by the equivalence relation generated by horizontal disks. We obtain a laminated space with a finer topology injecting into the space and covering the measure.

Besides the obvious and natural properties of this data, the two important aspects of the axioms are: (1) the vertical boundary covering property and (2) logarithmic bounds on the measures of subsets constructed from the boxes and the nested partitions.

Property (1), the vertical boundary covering property, was used in [11 and is a new property of Pesin boxes easily derived from the dynamics. It says that after $m$-negligible refinement of boxes, $T \times \partial(D i s k)$ is covered by the insides of other boxes.

Property (2) has two parts. The first part says that the log probability of a partition element at level $n$ containing $x$ is comparable to the log probability of a partition element at level $n$ containing $y m$-a.e. The second part says that we have the same comparison at $x$ and $y$, even after intersecting the $n$ th-level partition elements with any Borel collection of horizontal disks in a box. These log probability statements are due to Ledrappier and Young in [4 and explicated by Pesin and coauthors in 1 .

This paper is arranged as follows. In $\S 2$, we describe the data and axioms required to construct an abstract lamination with holonomy invariant information topology. In $\S 3$, we show how the work of Pesin, Ledrappier, and Young is used to construct such data satisfying the axioms. Together, $\S \S 2$ and 3 yield a proof of the main result which we now state.

Theorem 1.1. Let $M$ be a smooth compact Riemannian manifold without boundary, let $f$ be a $C^{1+\alpha}$-diffeomorphism from $M$ to itself, let $\mu$ be an $f$-invariant and ergodic Borel probability measure defined on the Borel algebra $\mathbb{B}$ in $M$ and $\mathcal{W}^{s / u}$ be the stable and unstable Pesin partitions of $M$, whose partition elements are the global stable and unstable manifolds through regular points of $f$. Then, if the $\mu$ entropy $h_{\mu}(f)$ is positive, there exist $f$-invariant stable and unstable laminations $\mathcal{L}^{s} \hookrightarrow \mathcal{W}^{s}$ and $\mathcal{L}^{u} \hookrightarrow \mathcal{W}^{u}$ whose global leaves are the stable and unstable global manifolds, which intersect transversely relative to the inherited leaf-wise Riemannian metrics at $\mu$-a.e. point $\in M$. Relative to the information topology, $\mathcal{L}^{s}$ and $\mathcal{L}^{u}$ satisfy the following additional properties.

(1) $\mathcal{L}^{u}\left(\mathcal{L}^{s}\right)$ is locally homeomorphic to open Pesin boxes of the form $T \times W_{r}^{u}$ $\left(T \times W_{r}^{s}\right)$, where $T$ is a Borel subset and $W_{r}^{u}\left(W_{r}^{s}\right)$ the local unstable (stable) manifold of some $x \in \Re$.

(2) The Riemannian metrics on global leaves are transversely measurable and continuous.

(3) The dynamics of $f$ are diffeomorphic and expanding (contracting) in the leaf direction, and measurable and continuous in the transverse direction.

(4) The injection $I^{u}: \mathcal{L}^{u} \hookrightarrow \mathcal{W}^{u}\left(I^{s}: \mathcal{L}^{s} \hookrightarrow \mathcal{W}^{s}\right)$ is continuous, commutes with the dynamics of $f$ and the image fills up the $\mu$-measure.

When the measure $\mu$ is hyperbolic in the sense that the Lyapunov exponents are nonzero, one can give a quantitative description of the lamination in terms of the transversal measure class. If one assumes further that the transversal measure class is holonomy invariant, then the stable and unstable laminations are measured laminations. The author has carried out the construction for this special setting in a separate paper (see [12] for the detailed discussion). 


\section{ACKNOWLEDGMENT}

We point out that the term information topology was suggested by Young and other experts in the field during Sullivan's talk at the conference at Princeton in honor of Mather's 60th birthday. This paper can be considered as the first part of a joint project with Dennis Sullivan on laminations for more general dynamical systems that are surjective on manifolds.

The author also expresses her sincere appreciation to the referee for his valuable comments.

\section{Abstract construction}

In this section, we explore the construction of laminations on an abstract probability space $(X, \mathbb{B}, m)$, where $\mathrm{X}$ is the underlying standard Borel space with a Borel probability measure $m$ defined on the Borel algebra $\mathbb{B}$.

\subsection{Data and axioms.}

Definition 2.1. A Borel subset $B \in \mathbb{B}$ is called a box if it is provided with a Borel isomorphism to the product $T \times \bar{D}$, where $T$ is a Borel subset and $\bar{D}$ is a closed $k$-disk in $\mathbb{R}^{k}$. The second factor $\{x\} \times \bar{D}$ is said to be the horizontal direction and the vertical factor $T_{x}=: T \times\{x\}$ is called the vertical or transversal direction of the box $B$.

The subset $T \times D$ is called the inside and $T \times \partial(D)$ is called the vertical boundary of the box, where $D$ is the interior of $\bar{D}$ and $\partial(D)$ is the topological boundary of $D$ in $\mathbb{R}^{k}$.

If $Y \subset B$ and $D_{y} \subset B$ is the horizontal disk containing $y \in Y$, then $B(Y)=$ : $\bigcup_{y \in Y} D_{y} \subset B$ is called the $B$-saturation of $Y$.

A subset $S$ of a box $B$ is called a $B$-saturated null subset if it is a $B$-saturation of $T^{\prime} \subset B$ with $m(S)=0$.

Note that boxes are closed under two operations: Vertical cutting of the form $T \times D_{1}, D_{1} \subset D$ and horizontal saturation.

We assume the following data for the construction.

\section{Data.}

(1) $X-$ A standard Borel space.

(2) $\mathbb{B}$ - The standard Borel structure on $X$.

(3) $m$ - A Borel probability measure with no atoms supported on $\mathbb{B}$.

(4) $\Gamma$ - A countable collection of boxes of the form $B=: T \times \bar{D}$.

(5) $\left\{\mathcal{P}_{n}\right\}_{n \geq 1}-$ An increasing sequence of countable partitions.

We assume the following axioms for the data.

Axiom 1. The countable family $\Gamma$ of boxes satisfies the following properties:

(a) For $m$ almost every point in the support of $m$, there are boxes $B(x) \in \Gamma$ containing $x$ of arbitrary small positive $m$-measures.

(b) Whenever two boxes $B_{1}=T_{1} \times D_{1}$ and $B_{2}=T_{2} \times D_{2}$ intersect, their overlap Borel isomorphisms are disk-preserving homeomorphisms in the horizontal direction. 
(c) The Vertical Boundary Covering Property: for each box $B=T \times \bar{D} \in \Gamma$, the vertical boundary can be covered by the insides of other boxes in $\Gamma$ after removing a $B$-saturated null set.

Axiom 2. The increasing sequence of countable partitions $\mathcal{P}_{1} \prec \mathcal{P}_{2} \prec \mathcal{P}_{3} \prec \cdots \prec$ $\mathcal{P}_{n} \ldots$ satisfies the following properties.

There exists $X^{\prime} \subset X$ of full $m$-measure such that:

(a) The measure $m\left(P_{n}(x)\right) \rightarrow 0$, as $n \rightarrow \infty$, for every $x \in X^{\prime}$, where $P_{n}(x)$ denotes the partition element in the $n$ th-level partition $\mathcal{P}_{n}$ containing $x$.

(b) $\left\{\mathcal{P}_{n}\right\}$ separates points in the sense that for every $x \neq y \in X^{\prime}, P_{n}(x) \neq P_{n}(y)$ eventually, as $n \rightarrow \infty$.

(c) For each pair $x, y \in X^{\prime}$,

$$
\varlimsup_{n \rightarrow \infty} \frac{\log \left(m\left(P_{n}(y)\right)\right)}{\log \left(m\left(P_{n}(x)\right)\right)}=O(1) .
$$

(d) Given any $m$-measurable subset $Y$ of a box $B=T \times \bar{D}$ in $\Gamma$ with $m(B(Y))>$ 0 , when $x, y \in X^{\prime}$ in the same horizontal disk,

$$
\varlimsup_{n \rightarrow \infty} \frac{\log \left(m\left(B(Y) \cap P_{n}(y)\right)\right)}{\log \left(m\left(B(Y) \cap P_{n}(x)\right)\right)}=O(1) .
$$

When the data satisfy the above axioms, we claim that there is a lamination structure injected in the underlying space.

Theorem 2.1 (Abstract construction). Suppose the Borel probability measure space $(X, \mathbb{B}, m)$ is provided with an increasing sequence of countable partitions $\left\{\mathcal{P}_{n}\right\}$ and a countable collection $\Gamma$ of boxes covering $X$ that satisfies the above axioms 1 and 2 . Then after removing countably many saturated null sets from $X$, there is a topology on the union $L$ of the refined boxes which satisfies the vertical boundary covering property exactly, so that these new boxes define a topological lamination $\mathcal{L}$.

The proof of Theorem 2.1 is derived by construction using the steps below.

\subsection{The space $L$.}

A. The m-density. We first borrow a useful concept from Shilov and Gurevich [10].

Definition 2.2. Let $E$ be a Borel subset of $X$ with a positive $m$-measure. A point $x \in X$ is called an $m$-density for $E$ with respect to the sequence of partitions $\left\{\mathcal{P}_{n}\right\}_{n \geq 1}$ if

$$
\lim _{n \rightarrow \infty} \frac{m\left(P_{n}(x) \cap E\right)}{m\left(P_{n}(x)\right)}=1
$$

provided that the limit exists.

Lemma 2.1 (Vitali-Lebesgue theorem). The subset of m-density points of $E$ has full m-measure of E.

Proof. See [10, pp. 209-215].

Definition 2.3. A point $x$ in a box $B=T \times \bar{D}$ is said to be a good point of $B$ if it is an $m$-density point of $B$ and the axioms 1(a) and 2 hold at $x$.

We observe the following facts. 
Lemma 2.2. For every box $B=T \times \bar{D}$ in $\Gamma, m(B)>0$, one can remove a saturated null set such that for every point $x \in B$ there is a good point $y$ of $B$ with $x \in D_{y}$.

Proof. We claim first that $m$-almost every point in a box $B \in \Gamma$ is a good point of $B$. In fact, the subset of $m$-density points of $B$ has full $m$-measure in $B$ and the subset of points at which the Axioms 1 (a) and 2 hold also has full $m$-measure in $B$. Hence, the intersection of the two subsets has full $m$-measure in $B$. Let $E$ be the subset of good points of $B$. Then, the $B$-saturation $B(E)=: \bigcup_{y \in E} D_{Y}$ is a sub-box of $B$ as desired with $m(B(E))=m(B)$.

\section{B. The saturation process.}

Proposition 2.1. After removing countably many saturated null sets, one can obtain a union of refined boxes $\bigcup B^{\prime}=L \subset X$ such that the vertical boundary covering property is true exactly for $L$. Namely, for each $B^{\prime}$, the vertical boundary $\partial_{T} B^{\prime}$ can be covered by the insides of other boxes.

Proof. Denote by $B_{0}$ any of the boxes in $\Gamma$. We apply Axiom 1(c) by removing $B_{0}$-saturated null sets to get a new set of boxes, denoted by $B_{1}$, so that the vertical boundary $\partial_{T} B_{1}$ of each $B_{1}$ is covered by $B_{0}$ exactly. Removing $B_{1}$-saturated null sets, we get a set of new boxes $B_{2}$, so that the vertical boundary $\partial_{T} B_{2}$ of each $B_{2}$ can be covered by $B_{1}$ exactly. This process can be done in, at most, countably many steps and then $B_{\infty}=\bigcap_{i=1}^{\infty} B_{i}$ covers exactly.

Let $L$ be the union of the insides of the countably many new boxes $B_{\infty}$ obtained form the above process. Then $L$ is as desired with $m(L)=1$.

Definition 2.4. A global leaf is an equivalence in $L$ generated by the relation: $x \sim$ $y$ if there are finitely many boxes $B_{i}=T_{i} \times D_{i} \in \Gamma$ such that for $i=1,2, \ldots, n-1$,

(a) $x=x_{1} \in B_{1}, y \in B_{n}$, and $x_{i} \in B_{i}$;

(b) $x_{i}$ and $x_{i+1}$ lie on the same "open" horizontal disk $D_{i}$ in a box $B_{i}$.

Proposition 2.2. A global leaf has the structure of a topological manifold that contains the "closure" of each "open" horizontal disk which intersects it.

Proof. For each refined box $B^{\prime}$ in $L$, Proposition 2.1 allows us to paste finitely many horizontal disks of other refined horizontal disks around every horizontal disk $D_{x}=\{x\} \times D$ in $B^{\prime}$.

We can then define a topology on every global leaf in $L$, using the sub-basis of all open sets of local horizontal disks. Then we claim that such a topology is Hausdorff. Indeed, if $x \neq y \in D_{x}$ are in the same horizontal disk, they can be separated by different open sets of the disk. Otherwise, if $x \sim y$, and $y$ not $\in D_{x}$, then $x$ and $y$ are separated by $D_{x} \backslash \overline{D_{y}}$ and $D_{y}$.

Observe also that Axiom 1(b) guarantees that overlap maps between horizontal disks on a global leaf, whenever they intersect, are homeomorphisms. The remaining statement in the proposition follows from Proposition 2.1.

Remark 2.1. To save notation, we denote the refined boxes by $B$, the countable collection of such boxes $B=T \times \bar{D}$ by $\Gamma$ and the union of all such refined boxes that Propositions 2.1 and 2.2 hold exactly by $L$ again. 


\section{Horizontal translations.}

Definition 2.5. Let $B=T \times D$ be a box in $\Gamma$. Take any two transversals $T_{x}=$ $T \times\{x\}$ and $T_{y}=T \times\{y\}$ of $B=T \times D$. Define the local horizontal translation in $B$ from $T_{x} \rightarrow T_{y}$ by sending points $x^{\prime} \in T_{x}$ along horizontal disks to points $y^{\prime} \in T_{y}$.

Let $x \sim y$ be two different points on the same global leaf $L(x)$. Then there are finitely many boxes $B_{i}=T_{i} \times D_{i} \in \Gamma$, such that for $i=1,2,3, \ldots, n-1$,

(a) $x \in B_{1}, y \in B_{n}$, and $x_{i+1} \in D_{i} \cap D_{i+1} \subset B_{i} \cap B_{i+1}$.

(b) The local horizontal translations $h_{i}$ of $T_{i}=T_{i} \times\left\{x_{i}\right\}$ to $T_{i+1}=T_{i+1} \times\left\{x_{i+1}\right\}$ are well defined within the boxes $B_{i}$.

The composition $h=h_{1} \circ h_{2} \circ \cdots \circ h_{n}$ defined on the subset

$$
T_{1} \cap h_{i}^{-1}\left(T_{2} \cap h_{2}^{-2}\left(T_{3} \cap \cdots T_{n-1} \cap h_{n}^{-1}\left(T_{n}\right)\right)\right)
$$

of the transversal $T_{x}=T_{1} \times\{x\}$ to a subset of the transversal $T_{y}=T_{n} \times\{y\}$ is a bijection and, whenever defined, is called a horizontal translation on $L$ from $T_{x}$ to $T_{y}$.

Remark 2.2. The domain of the horizontal translations at this stage may reduce to a single point. This is why we go further to define a topology.

\subsection{The information topology.}

A. Thickness. For each box $B=T \times \bar{D}$, we choose one transversal $T_{B}=T \times\{x\}$, such that $x \in B$ is a good point of $B$. Let $\Sigma=: \bigcup_{B \in \Gamma} T_{B}$ be the countable union of the transversals $T_{i}$ of boxes $B_{i} \in \Gamma$.

Definition 2.6. Let $B$ be a box in $\Gamma$ and let $Y$ be a Borel measurable subset of the chosen $\Sigma$ with $B(Y)>0$. $Y$ is said to be $m$-thick at a good point $y$ in $B$ if

$$
\frac{\log \left(m\left(B(Y) \cap P_{n}(y)\right)\right)}{\log \left(m\left(P_{n}(y)\right)\right)}=O(1) \text {, as } n \rightarrow \infty \text {. }
$$

The following statement shows that $m$-thickness is well-defined.

Proposition 2.3. If $x \sim x^{\prime}$ are both good points of the box $B \in \Gamma$, then $Y \subset T_{x}$ thick at $x$ goes to $Y^{\prime}=h(Y) \subset T_{Y}$ thick at $x^{\prime}$ under the horizontal translation $T_{x} \rightarrow T_{y}$ in the box $B$.

Proof. Notice that $B(Y)=B\left(Y^{\prime}\right)$ and the inequalities (1) and (2) in Axiom 2(c)(d) hold for both $x$ and $x^{\prime}$; we have

$$
\begin{aligned}
& \frac{\log \left(m\left(B\left(Y^{\prime}\right) \cap P_{n}\left(x^{\prime}\right)\right)\right)}{\log \left(m\left(P_{n}\left(x^{\prime}\right)\right)\right)} \\
& \quad=\frac{\log \left(m\left(B\left(Y^{\prime}\right) \cap P_{n}\left(x^{\prime}\right)\right)\right)}{\log \left(m\left(B(Y) \cap P_{n}(x)\right)\right)} \cdot \frac{\log \left(m\left(B(Y) \cap P_{n}(x)\right)\right)}{\log \left(m\left(P_{n}(x)\right)\right)} \cdot \frac{\log \left(m\left(P_{n}(x)\right)\right)}{\log \left(m\left(P_{n}\left(x^{\prime}\right)\right)\right)} \\
& \quad \sim C^{ \pm 2} \cdot \frac{\log \left(m\left(B(Y) \cap P_{n}(x)\right)\right)}{\log \left(m\left(P_{n}(x)\right)\right)}, \text { as } n \rightarrow \infty .
\end{aligned}
$$

Definition 2.7. If $y \in B$ is not a good point of $B$, then let $x \in B$ be a good point so that $y \in D_{x}$ and let $h$ be the horizontal translation within the box $B$ with $y=h(x)$. We say that $h(Y)$ is $m$-thick at $y$ if and only if $Y$ is $m$-thick at $x$ by Definition 2.6

Corollary 2.1. m-thickness is invariant under horizontal translations. 


\section{B. The $m$-topology on transversals.}

Definition 2.8. Let $\Sigma=\bigcup_{B \in \Gamma} T_{B}$ be chosen as above and let $Y$ be a Borel subset of $\Sigma$.

(1) $Y$ is said to be $m$-full at $y \in \Sigma \cap B$ if for any $F \subset \Sigma, m$-thick at $y$, then $Y \cap F$ is also $m$-thick at $y$.

(2) $Y$ is said to be $m$-open if $Y$ is $m$-full at every point $y \in Y$.

(3) Define $\emptyset$ to be $m$-open.

Lemma 2.3. The m-openness on transversals is invariant under horizontal translation.

Proof. This follows from the invariance of the $m$-thickness under horizontal translations.

Proposition 2.4. The m-openness satisfies the properties of a topology.

(i) If $Y_{1} \cap Y_{2} \neq \emptyset$ are two m-open subsets of $\Sigma$, then $Y_{1} \cap Y_{2}$ is also m-open.

(ii) If $Y_{\alpha} \subset \Sigma$ are $m$-open, so is $\bigcup_{\alpha} Y_{\alpha}$ in $\Sigma$.

(iii) $\Sigma=\bigcup_{B \in \Gamma} T_{B}$ is m-open.

Proof.

(i) For every $y \in Y \cap Y^{\prime}$, if $A \subset \Sigma$ is $m$-thick at $y$, then $Y \cap A$ is $m$-thick at $y$ because $Y$ is $m$-full at $y$. Since $Y^{\prime}$ is $m$-full at $y$, the intersection of $Y^{\prime} \cap(Y \cap A)$ is, in turn, $m$-thick at $y$. Thus, $\left(Y \cap Y^{\prime}\right)$ is $m$-full at $y$ and thus is $m$-open.

(ii) For every $y \in \bigcup Y_{\alpha}$, and any $A \subset \Sigma, m$-thick at $y$, there is an index $\alpha_{0}$ so that $y \in Y_{\alpha_{0}}$. Then $Y_{\alpha} \cap A$ is thick at $y$ and so is $\bigcup Y_{\alpha}$.

(iii) By the definition of $\Sigma=\bigcup T_{B}$, where $T_{B}=T \times\{x\}, x \in B$ is a good point of $B$, and by (ii), we need to show that every $T=T_{B}=T \times\{x\}$ is $m$-open.

(a) $T$ is $m$-thick at $x$ : Since $x$ is an $m$-density point of $B$ and $B(T)=B$, we have, for any $0<\varepsilon<1$ and $n$ sufficiently large, $(1-\varepsilon) m\left(P_{n}(x)\right) \leq$ $m\left(B(T) \cap P_{n}(x)\right) \leq(1+\varepsilon) m\left(P_{n}(x)\right)$. By taking the logarithm of the above inequality we have the assertion.

(b) $T$ is $m$-thick at every $y \in T$ : There exists a good point $y^{\prime} \in B$ such that $y=h\left(y^{\prime}\right)$ via a local horizontal translation defined from $T_{y^{\prime}}=T \times\left\{y^{\prime}\right\}$ to $T$ along the horizontal disks in the box $B$. By (a), $T_{y^{\prime}}$ is $m$-thick at $y^{\prime}$, and so is the $h$-image $T$ at $y$ by the invariance of the $m$-thickness under horizontal translations.

(c) $T$ is $m$-full at every point $y \in T$ : Indeed, $\forall A \subset T_{y}, m$-thick at $y \in T$, so is $A \cap T$ for $m\left(B(A \cap T) \cap P_{n}(y)\right)=m\left(B(A) \cap P_{n}(y)\right)$.

\section{The information topology.}

Definition 2.9. A box $B=T \times D$ is said to be fine-open if its transversal $T$ is $m$-open and the horizontal disk $D$ is open in the leaf topology.

Use all such open boxes to form a sub-basis and generate a topology on $L$. We call this topology the information topology. One can check that, equipped with the information topology, the space $L$ is Hausdorff. 
Lemma 2.4. The inside $B^{0}=T \times D \subset L$ of every refined box in $\Gamma$ is fine-open.

Proof. By the proof of Proposition 2.4(iii), the transversal of the refined box $B=$ $T \times \bar{D}$ is $m$-open and thus the inside $B^{0}=T \times D$ is fine-open by definition.

\subsection{The lamination on $L$.}

Proposition 2.5. Relative to the information-topology, $L$ is laminated by global leaves and satisfies the following properties:

(a) $L$ is a laminated space locally homeomorphic to fine-open boxes of the form $B=T \times D$.

(b) $L \hookrightarrow X$ has full $m$-measure of $X$.

(c) $L$ respects the Borel structure on $X$ (mod 0, r.w.t. $m$ ) in the sense that for every Borel subset $A$ in $\mathbb{B}$ with a positive $m$-measure, $\forall \epsilon>0$, there is a countable union of fine-open boxes of the form $B_{j}=T_{j} \times D_{j}$ such that $A \subset \bigcup_{j \geq 1} B_{j}$ and $m\left(\bigcup_{j \geq 1} B_{j} \backslash A\right)<\epsilon$.

Proof. By Lemma 2.4 the inside $B^{0}=T \times D$ of each refined box $B=T \times \bar{D}$ in $L$ is fine-open. As the countable union of all open boxes, $L$ is fine-open.

$L$ consists of global leaves, each of which has the structure of a topological manifold. We claim that the topological structures are transversely continuous relative to the information topology. In fact, the Borel isomorphism between the interior of every refined box to the product $T \times D$ becomes a continuous homeomorphism in the transversal direction relative to the transverse $m$-topology. Furthermore, whenever two fine open boxes intersect, the intersection is also fine-open and the overlap maps become continuous homeomorphisms transversely and horizontally. Therefore the topological structures on local disks are transversely continuous.

(a) For every point $x \in L$, there is a good point $y$ contained in a refined box $B(y)=T \times \bar{D}$ containing $x \in D_{y}$. Therefore, the fine-open box $B(y)^{0}=$ $T \times D$ can serve as a fine-open box neighborhood of $x$ in $L$.

(b) This follows from the construction of $L$ and Proposition 2.1.

(c) Given a Borel subset $A \in \mathbb{B}$ with $m(A)>0$ and $\varepsilon>0$, we denote by $A^{\prime}$ the subset of all the good points in $A$. For each $x \in A^{\prime}$, by Axiom 1(a) and Lemma 2.2, we have a refined box $B(x)=T(x) \times \overline{D(x)}$ that contains $x$ in the interior and has an arbitrary $m$-measure. Hence $A^{\prime} \subset \bigcup_{x \in A^{\prime}} B^{0}(x)$.

Notice that the union here is, at most, countable and thus we can select the $B(x)$ in such a way that the $m$-measure of the union is as required.

We point out that the horizontal translations from transversal to transversal on $L$ become homeomorphisms which are commonly known as holonomy homeomorphisms for the lamination. We have completed the proof of the main theorem 2.1.

\section{LAMINATION DATA FOR A DIFFEOMORPHISM}

In this section, we collect the lamination data for a system $(M, f, \mu, \mathbb{B})$, where $M$ is a smooth compact Riemannian manifold without boundary, $f$ is a $C^{1+\alpha_{-}}$ diffeomorphism of $M$ onto itself, for $0<\alpha \leq 1$, and $\mu$ is an $f$-invariant and ergodic 
Borel probability measure defined on the Borel algebra $\mathbb{B}$. We assume that the entropy $h(f)$ with respect to $\mu$ is positive.

3.1. Existing data. Let us begin by recalling some terms, facts, and notations in Pesin theory. For $x \in M$, let $T_{x} M$ denote the tangent space to $M$ at $x$. The point $x$ is said to be regular if there exist measurable functions $k(x), \lambda_{1}(x)>$ $\lambda_{2}(x)>\cdots>\lambda_{k(x)}(x)$, and a measurable decomposition of the tangent space $E(x)=: \bigoplus_{1 \leq i \leq k} E_{i}(x)$, such that for every non-zero tangent vector $v \in E_{i}(x)$,

$$
\lim _{n \rightarrow \infty} \frac{1}{n} \log \left\|D f^{n}(x) v\right\|=\lambda_{i}(x)
$$

and

$$
\lim _{n \rightarrow \infty} \frac{1}{n} \log \left|\operatorname{Jac}\left(D f^{n}(x)\right)\right|=\sum_{i=1}^{k(x)} \lambda_{i}(x) \operatorname{dim}\left(E_{i}(x)\right), \quad i=1, \ldots, k(x) .
$$

By a theorem of Oseledec ([6]), the set $\Re$ of regular points of $f$ is of full $\mu$-measure. The number $\lambda_{i}(x)$ is called the ith-Lyapunov exponent of $f$ at $x, i=1, \ldots, k(x)$. The functions $x \rightarrow k(x), \lambda_{i}(x)$, and $\operatorname{dim} E_{i}(x)$ are invariant along orbits, and thus constants $\mu$-almost everywhere since $\mu$ is ergodic. Define

$$
E^{s}(x)=\bigoplus_{\lambda_{i}<0} E_{i}(x), E^{c}(x)=E_{i_{0}}(x), \text { where } \lambda_{i_{0}}(x)=0, E^{u}(x)=\bigoplus_{\lambda_{i}>0} E_{i}(x) .
$$

Let $\rho$ be the Riemannian metric on $M$ and set

$$
W^{u}(x)=\left\{y \in M: \limsup _{n \rightarrow \infty} \frac{1}{n} \log \rho\left(f^{-n}(x), f^{-n}(y)\right)<0\right\}
$$

and

$$
W^{s}(x)=\left\{y \in M: \limsup _{n \rightarrow \infty} \frac{1}{n} \log \rho\left(f^{n}(x), f^{n}(y)\right)<0\right\} .
$$

Define $W_{r}^{u}(x)$ (and $W_{r}^{s}(x)$ ) to be the component of $W^{u}(x)$ (and $W^{s}(x)$ ), containing $x$, in the $r$-disk neighborhood of $x$. Then, carrying the inherited Riemannian structure from $M, W^{u}(x)$, tangent at $x$ to $E^{u}(x)$ (and $W^{s}(x)$ to $E^{s}(x)$ ), are $C^{1+\alpha_{-}}$ immersed sub-manifolds. The sets $W^{u}(x), W^{s}(x), W_{r}^{u}(x)$, and $W_{r}^{s}(x)$ are known as the global and local unstable and stable manifolds through $x \in \Re$, respectively. The radius $r(x)$ of the local unstable and stable manifolds is $\mu$-measurable and bounded above and below exponentially along orbits. We denote by

$$
\lambda=\min \left\{\left|\lambda_{i}\right|\right\}, \quad u=\operatorname{dim} E^{u}, \quad c=E^{c}(x), \quad s=\operatorname{dim} E^{s},
$$

and assume that $u>0$.

We point out that the collection of global stable and unstable manifolds $\mathcal{W}^{s}=$ $\left\{W^{s}(x): x \in \Re\right\}$ and $\mathcal{W}^{u}=\left\{W^{u}(x): x \in \Re\right\}$ form the so-called Pesin stable and unstable partitions of $M$, which are commonly referred to as the "stable and unstable foliations" of $f$ on $M$. They do not, however, form real foliations or laminations relative to the usual topology on $M$. In this section, we work with the unstable partition $\mathcal{W}^{u}$ and collect the data required for a true lamination. 


\section{Existing data.}

- The underlying space $X=: M$ - a boundary-free smooth compact Riemannian manifold provided with a $C^{1+\alpha}$-diffeomorphism $f$.

- $\mathbb{B}$ - the standard Borel structure on $M$.

- $\mu$ - an $f$-invariant and ergodic Borel probability measure with no atoms.

- $\mathcal{P}_{n}$ - the increasing sequence of countable partitions: Let $\mathcal{P}$ be the countable partition of finite entropy constructed by Ledrappier and Young (4]) and let $\mathcal{P}_{n}=: \bigvee_{j=-n}^{n} f^{-j}(\mathcal{P})$ be the increasing sequence of refined partitions under the dynamics.

3.2. The countable collection of Pesin boxes. In this subsection, we construct an $f$-invariant countable collection $\Gamma$ of boxes, which are called Pesin boxes in the sequel.

Proposition 3.1. There exists an $f$-invariant countable family $\Gamma$ of Pesin boxes of the form $B=: T \times W_{r}^{u}$ whose union covers the regular set $\Re$ mod zero relative to $\mu$.

We give the proof by construction.

A. The countable decomposition of the regular set $\Re$. By standard measure theory, there exists a countable decomposition of the regular set $\Re=\bigcup_{m \geq 1} \Re_{m}$ such that for every $m \in \mathbb{N}$, the subset $\Re_{m}$ satisfies the following properties:

$\forall 0<\epsilon<1$, there exist constants $0<r_{m}<1$ and $C_{m}>1$, independent of $x \in \Re_{m}$, such that:

(i) The radius $r(x)$ of $W_{r(x)}^{u}(x)$ and $W_{r(x)}^{s}(x)$ is $\geq r$ and $\mu_{p}^{u}\left(W^{u}(x, r)\right) \geq$ $\frac{1}{2^{m}}$ and $\mu_{p}^{s}\left(W^{s}(x, r)\right) \geq \frac{1}{2^{m}}$ for $r=\frac{r_{m}}{2}>0$.

(ii) The Riemannian metrics on the local stable and unstable manifolds of radius $r$ and the maps $x \mapsto W_{r}^{u}(x)$ and $x \mapsto W_{r}^{s}(x)$ are uniformly continuous on $\Re_{m}$.

(iii) For $y \in W_{r}^{u}(x)$,

$$
\begin{aligned}
& \rho\left(f^{-n} x, f^{-n} y\right) \leq C e^{-n \lambda}, \quad y \in W_{r}^{s}(x), \quad \rho\left(f^{n} x, f^{n} y\right) \leq C e^{-n \lambda}, \\
& \quad \text { and } \\
& \quad \sin \left(\angle\left(E^{\alpha}(x) E^{\beta}(x)\right)\right) \geq C^{-1}>0, \quad \alpha, \beta \in\{s, c, u\} .
\end{aligned}
$$

Choose $m \in \mathbb{N}$ and $C_{m}$ sufficiently large that $\mu\left(\Re \backslash \Re_{m}\right)<\frac{\epsilon}{2}$ and set

$$
\Lambda=\left\{q \in \Re: f^{n}(q) \in \Re_{m} \text { for infinitely many } n \text { 's }\right\} .
$$

Then $\Lambda$ is $f$-invariant and hence $\mu(\Lambda)=1$. We denote the set $\Re_{m} \cap \Lambda$, again, by $\Re_{m}$.

B. The Pesin boxes. For any $\mu$-measurable subset $E$, using the increasing sequence of countable partitions $\left\{\mathcal{P}_{n}\right\}$, we obtain a full $\mu$-measure subset of density points of $E$. We point out that the Vitali-Lebesgue theorem (see Lemma 2.1) also guarantees that $\mu$-almost every point $x \in E$ is a Lebesgue-density point for $E$ in the sense that $\lim _{r \rightarrow 0} \frac{\mu((D x, r) \cap E)}{\mu((D x, r))}=1$, where $D(x, r)$ is a disk-neighborhood of $x$ of radius $r>0$ centered at $x$. Without loss of generality, in the sequel, we will take 
the density points of $E$ to be in the intersection of the two types without referring to either Lebesgue-density or $\mu$-density.

Given a Borel subset $A \subset \Re_{m}$ of diameter $<r$ and given any number $\delta>0$, we denote by $W^{u}(x, \delta)$ the component of $W^{u}(x)$ in the $\delta$-neighborhood $N(A, \delta)$ of $A$, containing $x . W^{u}(x, \delta)$ can also be viewed as a local unstable manifold of $x$. Now for a fixed $0<\varepsilon<\frac{\lambda}{4}$, let $x_{0} \in \Re_{m}$ be a density point of $\Re_{m}$ and let $A=\Re_{m} \cap D\left(x_{0}, r\right)$, where $0<r<\frac{r_{m}}{2}$ is sufficiently small that $\mu(A)=\left(1-\frac{\varepsilon}{2}\right) \mu\left(D\left(x_{0}, r\right)\right)$;

$$
C_{m}^{-1} e^{-n(h+\varepsilon)} \leq \mu^{u / s}\left(W^{u / s}(x, r) \cap P_{n}(x)\right) \leq C^{m} e^{-n(h-\varepsilon)}
$$

holds for all $x \in A$ and all $n \geq 0$, where $\mu^{u}$ and $\mu^{s}$ denote the conditional measures of $\mu$ associated to the partitions $\xi^{u}$ and $\xi^{s}$ subordinate to $\mathcal{W}^{u}$ and $\mathcal{W}^{s}$, and $h$ is the Kolmogorov-Sinai entropy of $f$ (see [4] for the definition and more properties of the partitions $\xi^{u}$ and $\xi^{s}$ ).

Definition 3.1. Define

$$
B=B\left(x_{0}\right)=: \bigcup_{x \in A} \overline{W^{u}(x, r)}
$$

and call $B$ a Pesin box for the unstable partition $\mathcal{W}^{u}$, of radius $r$ generated by $A$, where $\overline{W^{u}(x, r)}$ is the closed local unstable manifold of $x$ in the $r_{0}$-neighborhood of $A$ with respect to the relative topology on the global unstable manifold $W^{u}(x)$.

Such Pesin boxes possess the following properties.

Lemma 3.1. The Pesin box $B(A)$ is homeomorphic to the product $T \times \overline{W^{u}(x, r)}$.

Proof. According to Ledrappier and Young [4 p. 528], there exists a specified smooth sub-manifold $\Sigma$ which intersects every local unstable disk $W^{u}(x, r) \subset B$ transversely at a unique point and satisfies:

(a) $\Sigma$ can be viewed as the graph of a smooth function from a small disk in $\mathbb{R}^{c+s}$ to a small disk in $\mathbb{R}^{u}$ with slope $\leq \frac{1}{3}$; and

(b) for every $x \in A$, there exists $\Sigma(x)$ homeomorphic to $\Sigma$ such that $W^{s}(x, r) \subset$ $\Sigma(x)$.

Lemma 3.2. Every $\Re_{m}$ can be covered by countably many Pesin boxes:

$$
\Re_{m} \subset \bigcup_{i \geq 1} B\left(A_{i}\right)
$$

of the form $T_{i} \times W_{r}^{u}\left(x_{i}\right)$ as defined in Lemma 3.1, around a $\mu$-density point $x_{i}$. Therefore, the regular set $\Re$ can be covered by countably many Pesin boxes of arbitrarily small $\mu$-measure (mod zero relative to $\mu$ ).

Proof. Fix $r=\frac{r_{m}}{2} \leq e^{-n_{0}(\lambda+\varepsilon)}$. The set $\Re_{m}$ can be covered by countably many $A_{i}=\Re_{m} \cap D\left(x_{i}, \frac{r}{2}\right)$ with $x_{i} \in \Re_{m}$. Let $B\left(A_{i}\right)$ be the Pesin box of radius $r$ generated by $A_{i}, i=1,2, \ldots$. Then $\Re_{m} \subset \bigcup_{i \geq 1} B\left(A_{i}\right)$.

We denote by $\Gamma_{0}$ the countable collection of Pesin boxes whose union covers $\Re$ (mod zero relative to $\mu$ ). 
Definition 3.2. A $\mu$-measurable subset $B$ is called a Pesin box in the general sense for the unstable partition $\mathcal{W}^{u}$ if $B$ is homeomorphic to the product of the form $T(x) \times \overline{W_{r}^{u}(x)}$, where $T(x)$ is $\mu$-measurable so that $T(x) \cap \xi^{s}(x)$ is $\mu^{s}$-measurable, and $\overline{W_{r}^{u}(x)}$ the closure (in the leaf topology) of the local unstable manifold for some point $x \in B \cap \Re$.

For any subset $Y$ of a Pesin box $B=T \times \overline{W_{r}^{u}}$, the subset $B(Y)=: \bigcup_{y \in Y} \overline{W^{u}(y, r)}$

of $B$ is called the $B$-saturation of $Y$, where $\overline{W^{u}(y, r)} \subset B$ is the closure (in the leaf topology) of the local unstable leaf in $B$ containing $y$.

One can also check the following fact.

Lemma 3.3. Let $B=B\left(A_{i}\right) \in \Gamma_{0}$ be the Pesin box generated by $A_{i}=\Re_{m} \cap$ $D\left(x_{i}, r\right) \ni x_{i}$. Then $f(B)$ is also a Pesin box homeomorphic to the product $f\left(T_{i}\right) \times$ $f\left(D\left(x_{i}, r\right)\right)$.

Proof. This follows because $f$ is a $C^{1+\alpha}$-diffeomorphism $f$ on $M$.

We define $\Gamma$ to be the countable collection of all Pesin boxes of the form $T_{i} \times W_{i}^{u}$ in $\Gamma_{0}$, together with their forward and backward images under the dynamics of $f$. Then $\Gamma$ is $f$-invariant and the union of the Pesin boxes in $\Gamma$ covers $\Re(\bmod 0$ rel. $\mu)$.

This completes the proof of Proposition 3.1.

\section{THE AXIOMS FOR A DIFFEOMORPHISM}

We show, in this section, that the Pesin boxes constructed in the previous section and the sequence of the partitions $\left\{\mathcal{P}_{n}=: \bigvee_{k=-n}^{n} f^{-k}(\mathcal{P})\right\}$ satisfy Axioms 1 and 2 stated in Section 2 ,

\section{1. $\Gamma$ satisfies Axiom 1.}

Proposition 4.1. Let $\Gamma$ be the countable collection of Pesin boxes constructed in the previous section.

(a) For every density point $x_{0} \in \Re_{m}$, there exists a decreasing sequence $r_{m_{i}} \rightarrow$ 0 , as $m_{i} \rightarrow \infty$, and Pesin boxes $B_{m_{i}}\left(x_{0}\right)=T\left(x_{0}\right) \times W_{r_{m_{i}}}^{u}\left(x_{0}\right) \in \Gamma$ of radii $r_{m_{i}}$ such that the union of the Pesin boxes $B_{m_{i}}\left(x_{0}\right)$ covers the local stable manifold $W_{r_{m}}^{s}\left(x_{0}\right)$ up to a $\mu_{x_{0}}^{s}$-measure zero. In particular, Axiom 1(a) holds for $\Gamma$.

(b) Whenever two Pesin boxes in $\Gamma$ intersect, the overlap maps are Borel measurable in the transversal direction and $C^{1+\alpha}$ smooth in the disk direction. Furthermore, the Riemannian structure on the local unstable manifolds is uniformly continuous in the transversal direction.

(c) [Vertical Boundary Covering Property]. For every Pesin box $B=T \times W_{r}^{u} \in$ $\Gamma$, the vertical boundary $\partial_{T} B=T \times \partial W_{r}^{u}$ of $B$ can be covered by other Pesin boxes in $\Gamma$, up to a B-saturated null subset of the form $Y \times \overline{W_{r}^{u}} \subset B$ for some $Y \subset T$.

Proof. (a) From the construction of $\Re_{m}$, one observes that the regular set $\Re=\bigcup \Re_{m}$ is an increasing union $\Re_{1} \subset \cdots \subset \Re_{m} \subset \ldots$ Then for every density point $x_{0} \in \Re_{m}$, one can shrink the radii $r_{m}$ of the unstable and stable manifolds uniformly with $r_{m} \rightarrow 0$, as $m \rightarrow \infty$ such that $A_{m}\left(x_{0}\right)=\Re_{m} \cap D\left(x_{0}, r_{m}\right)$ covers a bigger proportion of the $\mu$-measure of $D\left(x_{0}, r_{m}\right)$ and the transversal $T_{m} \cap W^{s}\left(x_{0}, r_{m}\right)$ covers a bigger 
proportion of the $\mu^{s}$-measure of $W^{s}\left(x_{0}, r_{m}\right)$, as $m \rightarrow \infty$. In particular, Axiom 1(a) holds.

(b) Since the dimension $u$ of the unstable manifold $W^{u}(x)$ is almost constant for $x \in \Re$, we may assume that it is constant at every point $x \in \Re_{m}$. From the definition of Pesin boxes $B\left(x_{i}\right)$ generated by $A_{i}=\Re_{m} \cap D\left(x_{i}, r_{m}\right)$, the horizontal disks are in fact the local unstable manifolds through the points in $A_{i}$ which are $C^{1}$-close in the transversal direction. Whenever two such Pesin boxes intersect, the overlap maps can be chosen as overlap maps of the local unstable manifolds. These overlaps are Borel in the transversal direction. Moreover, by the definition of $\Re_{m}$, the Riemannian structures of the local unstable disks in $B\left(A_{i}\right)$ are uniformly continuous in the transversal direction. Such properties are clearly preserved by the dynamics of $f$ in both the forward and backward iterations.

(c) Without loss of generality, we may assume that $B=B\left(A_{i}\right)$ is the Pesin box generated by $A_{i}=\Re_{m} \cap D\left(x_{i}, r\right)$, for some density point $x_{i} \in \Re_{m}$ with $\mu(B) \geq$ $\mu\left(A_{i}\right)>(1-\varepsilon) \mu D\left(x_{i}, r\right)$. Choose $E \subset T$, such that $B(E)=: E \times \overline{W^{u}\left(x_{i}, r\right)} \subset B$ has positive $\mu$-measure. Since $\mu$ is ergodic, $f^{-n}(x)$ returns to $B(E)$ for infinitely many $n$ for $\mu$-a.e. $x \in B(E)$. Choose $n$ large enough so that $C e^{-n \lambda}<r$. It is evident that $F=: f^{-n}(B(E)) \cap B(E)$ has positive $\mu$-measure. The local unstable disks of $f^{-n}(B(E))$ are compactly contained in the local unstable disks of $B(E)$ whenever they intersect. Thus $f^{n}\left(f^{-n}(B(E)) \cap B(E)\right)$ is compactly contained in $\left(f^{n}(B) \cap B\right)$ in the leaf direction. That is, the part of the vertical boundary $\partial_{T} B(F) \subset E \times \partial_{T}\left(W^{u}\left(x_{i}, r\right)\right)$ can be covered by the Pesin box $f^{n}(B) \in \Gamma$.

Continue this process to cover the remaining part of the vertical boundary $B(E)$ by other Pesin boxes of form $f^{\ell}\left(B_{j}\right)$ for some $B_{j} \in \Gamma$, by deleting the vertical boundary of a $B$-saturated null subset of the form $Y^{\prime} \times W^{u}\left(x_{i}, r\right)$. This covering process is at most countable.

4.2. The sequence of partitions $\left\{\mathcal{P}_{n}\right\}_{n \geq 1}$ satisfies Axiom 2. We may require that the special sequence $\left\{\mathcal{P}_{n}\right\}$ of countable partitions of Ledrappier and Young refine every Pesin box in the countable collection $\Gamma$.

Proposition 4.2. The sequence $\left\{\mathcal{P}_{n}\right\}$ of countable partitions satisfies all the properties stated in Axiom 2 (see Section [2.1).

Proof. For the special countable partition $\mathcal{P}$ of finite entropy, according to Ledrappier and Young, given any $\varepsilon>0$, there exists a subset $\Lambda \subset \Re$, a constant $C>1$, and an integer $n_{0}$, independent of $x \in \Lambda$, such that for every $x \in \Lambda$ and $n \geq n_{0}$.

(i) $P_{n}(x) \subset D\left(x, 2 e^{-n}\right)$ (see [4 pp. 568-569]).

(ii) $P_{n}(x) \cap \xi^{u}(x) \subset D^{u}\left(x, e^{-n(\lambda-\varepsilon)}\right)$ (see Lemma 10.2(3) [4, p. 560] for $i=u$ ).

(iii) $C^{-1} e^{-2 n(h+\varepsilon)} \leq \mu\left(P_{n}(x)\right) \leq C e^{-2 n(h-\varepsilon)}$ (see Estimate (4) [4, p. 568]).

(iv) $C^{-1} e^{-n(h+\varepsilon)} \leq \mu^{u}\left(P_{n}(x) \cap \xi^{u}(x)\right) \leq C e^{-n(h-\varepsilon)}$ (the estimate from below is Lemma 10.2(4), for $i=u$ [4, p. 560]. For the estimate from above, one knows that $h_{\mu}=h$ (see Corollary 10.2(2) [4, p. 543]).

We may assume that for each $m$, every point $x$ is a density point in $\Re_{m}$, and the above inequalities hold for all $x \in \Re_{m}$. Otherwise, we can take a smaller version of 
$\Re_{m}$. Let $X^{\prime}=\bigcup \Re_{m}$. Then we obtain the following.

Axiom 2(a)-(b). It follows from (i) and (ii) above that $\operatorname{diam}\left(P_{n}(x)\right) \rightarrow 0$ and $\mu\left(P_{n}(x)\right) \leq C e^{-2 n(h+\varepsilon)} \rightarrow 0$, for every $x \in X^{\prime}$, as $n \rightarrow \infty$.

Axiom 2(c). One can obtain, from (ii) above, that $\frac{\log \left(\mu\left(P_{n}(x)\right)\right)}{\log \left(\mu\left(P_{n}(y)\right)\right.} \leq C^{\prime} \frac{h+\varepsilon}{h-\varepsilon}$, for some $C^{\prime}$ independent of all $x$ and $y \in X^{\prime}$.

Axiom 2(d). Let $B=B\left(A_{i}\right)=T_{i} \times W_{r}^{u}\left(x_{i}\right)$ be the inside of a Pesin box, generated by $A_{i}=\Re_{m} \cap D\left(x_{i}, r\right)$ for some point $x_{i} \in \Re_{m}$, in the countable collection $\Gamma$ of Pesin boxes constructed in the previous section. We observe the following fact and give its proof later.

Lemma 4.1. For any $Y \subset T$ and $z \in B$, the $B$-saturation $B(Y)$ satisfies $B(Y) \cap$ $P(z)=\bigcup_{q \in B(Y)}\left(D_{q} \cap P_{n}(z)\right)$.

Now, for any $Y \subset T$ and $z \in\{x, y\}$, let $Y_{z}=B(Y) \cap T_{z}$. Then $B(Y)=B\left(Y_{z}\right)$. Use a generalized Fubini result to "disintegrate" the measure $\mu$ as follows:

$$
\mu(E)=\int \mu_{y}^{u}\left(E \cap D_{y}\right) d \eta,
$$

where $\eta$ is the "push forward" measure on $B(E)$ defined by $\eta(E)=\mu(B(E))$ for any measurable subset $E \subset B$. Then

$$
\begin{aligned}
\mu\left(B\left(Y \cap P_{n}(x)\right)\right) & =\mu\left(\bigcup_{q \in B(Y)} D_{q} \cap P_{n}(x)\right) \\
& =\int_{B(Y)} \mu_{z}^{u}\left(W^{u}(z, r) \cap P_{n}(z)\right) d \eta \\
& \leq C e^{-n_{0}(h-\varepsilon)} \cdot \mu\left(T_{x} \cap P_{n}(x)\right) .
\end{aligned}
$$

Notice again that the partition $\mathcal{P}_{n}$ refines the Pesin box $B, P_{n}(q)=P_{n}(x)$ for every $q \in B(Y) \cap P_{n}(x)$, and denote $\mu_{q}^{u}\left(P_{n}(x)\right)=\mu_{q}^{u}\left(P_{n}(q) \cap \xi^{u}(q)\right)$. Using the above estimate (ii), we obtain the inequalities

$$
\begin{aligned}
\mu\left(B\left(Y_{x}\right) \cap P_{n}(x)\right) & =\int_{B\left(Y_{x}\right)} \mu_{q}^{u}\left(W^{u}(q, r) \cap P_{n}(x)\right) d \eta \\
& \leq \eta\left(B\left(Y_{x}\right)\right) \cdot C e^{-n(h-\varepsilon)}=C \cdot \mu\left(B\left(Y_{x}\right)\right) \cdot e^{-n(h-\varepsilon)}
\end{aligned}
$$

and

$$
\begin{aligned}
\mu\left(B\left(Y_{y}\right) \cap P_{n}(y)\right) & =\int_{B\left(Y_{y}\right)} \mu_{q}^{u}\left(W^{u}(q, r) \cap P_{n}(y)\right) d \eta \\
& \geq \eta\left(B\left(Y_{y}\right)\right) \cdot C e^{-n(h+\varepsilon)}=C \cdot \mu\left(B\left(Y_{y}\right)\right) \cdot e^{-n(h+\varepsilon)} .
\end{aligned}
$$

Combining these two inequalities, we obtain Axiom 2(c).

Proof of Lemma 4.1. For every $p \in B(Y) \cap P_{n}(z)$, there is a $q \in Y$ such that $p \in D_{q}$ and $p \in P_{n}(x)$. Hence, $p \in D_{q} \cap P_{n}(z)$ and then $p \in \bigcup D_{q} \cap P_{n}(z)$. On the other hand, for every $p$ contained in the union $\bigcup D_{q} \cap P_{n}(z)$, there is a $q \in B(Y)$ such that $p \in D_{y} \cap P_{n}(z)$, which implies that $p \in B(Y) \cap P_{n}(z)$. 
Applying Proposition 2.1] we obtain a subset $L^{u} \subset \mathcal{W}^{u}$ of global unstable manifolds which can be covered by the insides of refined Pesin boxes in $\in \Gamma$. Introducing the $\mu$-topology on transversals and the information topology on $M$, we have a true lamination $\mathcal{L}^{u}$ that satisfies the following additional properties.

Proposition 4.3. The lamination $\mathcal{L}^{u}$ obtained above is $f$-invariant and, relative to the information topology, it possesses the following additional properties.

(a) The Riemannian structure on global leaves is transversely measurable and continuous.

(b) The dynamics of $f$ is an expanding diffeomorphism in the leaf direction and continuous in the transversal direction.

(c) The image of the injection $I^{u}: \mathcal{L}^{u} \hookrightarrow \mathcal{W}^{u}$ fills up the $\mu$-measure.

Proof. This is evident from the construction of the Pesin boxes in $\Gamma$.

Remark 4.1. Replacing $u$ by $s$ (or $s$ by $u$ ), unstable by stable (or stable by unstable), we obtain an $f$-invariant lamination $\mathcal{L}^{s}$ continuously injected in the stable partition $\mathcal{W}^{s}$ so that the image of the injection intersects $\mathcal{W}^{s}$ in a full $\mu$-measure. The restriction of $f$ to $\mathcal{L}^{s}$ is a contracting diffeomorphism in the leaf direction.

Remark 4.2. From the construction, one can derive that $\mathcal{L}^{s}$ and $\mathcal{L}^{u}$ intersect transversely at every intersection point and this intersection fills up the $\mu$-measure of the regular set.

Proposition 4.4. The dynamical triple $\left(\mathcal{L}^{u}, \mathcal{L}^{s}, f\right)$ is well defined by the properties listed in Theorem 1.1, up to measure theoretic isomorphisms which are smooth in one of the leaf directions, and measure preserving and continuous in the transversal direction, respectively.

Proof. Suppose that there is another triple $\left(\mathcal{L}^{\prime u}, \mathcal{L}^{\prime s}, f\right)$ satisfying the properties stated in the main theorem. To save notation, we let $\mathcal{L}^{\prime}=\mathcal{L}^{\prime}$. . Then there is a continuous injection $J: \mathcal{L}^{\prime} \rightarrow M$ so that the image $J\left(\mathcal{L}^{\prime}\right)$, contained in $\mathcal{W}$, has full $\mu$-measure. Take the intersection $S=J\left(\mathcal{L}^{\prime}\right) \cap \mathcal{L}$. Then $S$ has full $\mu$-measure and so is the $f$-invariant saturation $\bar{S}=\bigcup f^{n}(S)$. Moreover, the composite map $\Phi=: J^{-1} \circ I: I^{-1}(\bar{S}) \rightarrow J^{-1}(\bar{S})$ is smooth in the leaf direction and continuous in the transversal direction for the information topology.

We complete the proof of Theorem 1.1.

\section{The nested FAMily of UnStABle LAMinations}

Now for every $i \in\{1,2, \ldots, u\}$ with $\lambda_{i}>0$, define

$$
W^{i}(x)=\left\{y \in M: \limsup _{n \rightarrow \infty} \frac{1}{n} \log \left(\rho\left(f^{-n}(x), f^{-n}(y)\right)\right) \leq-\lambda_{i}\right\} .
$$

Then $W^{i}(x)$ is a $C^{2}\left(\Sigma_{j \leq i} \operatorname{dim} E_{j}\right)$ dimensional immersed sub-manifold of $M$ tangent to $\bigoplus_{j \leq i} E_{i}(x)$ at $x \in \Re$. It is known as the $i$ th-unstable manifold of $f$ at $x$. The collection $\mathcal{W}^{i}=\left\{W^{i}(x): x \in \Re\right\}$ is sometimes referred to as the $i$ thunstable "foliation" on $M$. Using this language, one has a nested family of "a.e.foliations": $\mathcal{W}^{1} \subset \mathcal{W}^{2} \subset \cdots \subset \mathcal{W}^{u}$ corresponding to the distinct Lyapunov exponents $\lambda_{1}>\lambda_{2}>\cdots>\lambda_{u}$ of $f$.

If the conditional entropy $h_{i}$ is positive for each $i$, we may employ the same techniques and obtain a nested family of true laminations. 
Theorem 5.1. Let $(m, f, \mu, \mathbb{B})$ be the Pesin system as in Theorem 1.1. If the conditional entropy $h_{i}$ is positive for each $i$, then there exists a nested family of $f$-invariant laminations $\mathcal{L}^{i} \hookrightarrow \mathcal{W}^{i}$ with $\mathcal{L}^{1} \subset \mathcal{L}^{2} \subset \cdots \subset \mathcal{L}^{u}$ which satisfies the following additional properties.

(1) Each $\mathcal{L}^{i}$ is locally homeomorphic to the Pesin box product chart of the form $B=T \times W^{i}(x, r)$ whose global leaves are the $i$ th-unstable manifolds $W^{i}(x)$ through points $x \in \mathcal{L}^{i}$.

(2) The Riemannian metrics on global leaves are continuous in the transverse direction.

(3) The dynamics of $f$ is $C^{1+\alpha}$-diffeomorphic and expanding in the leaf direction and continuous in the transverse direction.

(4) The inclusion $I^{i}: \mathcal{L}^{i} \hookrightarrow \mathcal{W}^{i}$ is continuous and its image fills up the $\mu$ measure of $\mathcal{W}^{i}$.

Proof. The proof is similar to that of Theorem 1.1 when we use our new ingredients of the Pesin boxes and the entropy topology. By changing the symbols from $u$ to $i$ or $i-1$, we obtain a nested family of true laminations.

\section{REFERENCES}

[1] L. Barreire, Ya. Pesin and J. Schmeling, Dimension and Product Structure of Hyperbolic Measures, Annals of Math. (149) (1999), 755-783. MR 2000f:37027

[2] J. P. Eckman and D. Ruelle, Ergodic Theory of Chaos and Strange Attractors, Rev. Modern Phys. (57) (1885), 617-656. MR 87d:58083a

[3] A. Fathi, M.-R. Herman and J.-C. Yoccoz, A proof of Pesin's stable manifold theorem, Geometric Dynamics (J. Palis, Jr., Ed.), Lecture Notes in Math., Vol. 1007, Springer-Verlag, (1983), 177-215. MR 85j:58122

[4] F. Ledrappier and L. S. Young, The Metric Entropy of Diffeomorphisms, Part I: Characterization of Measures Satisfying Pesinś Entropy Formula, and Part II: Relations between Entropy, Exponents and Dimension, Annals of Math. (122) (1985), 509-539 and 540-574.

[5] R. Mañè, A proof of Pesin's formula, Ergodic Theory and Dynamical Systems (1) (1981), 95-102. MR 83b:58042

[6] V. I. Oseledec, Multiplicative ergodic theorem, Lyapunov characteristic numbers for dynamical systems, Trans. Mosc. Math. Soc. (19) (198), 197-221.

[7] Ya. B. Pesin, Families of invariant manifolds corresponding to non-zero characteristic exponents, English Transl., Math USSR-Izv. (10) (1976), 1261-1305.

[8] _ Characteristic Lyapunov exponents and smooth ergodic theory, English transl., Russian Math. Surveys (32) (1977), 55-114.

[9] C. Pugh and M. Shub, Ergodic Attractors, Trans. of AMS, Vol. 312 (1) (1989), 1-54. MR 90h:58057

[10] G. E. Shilov and B. L. Gurevich, Translated by R. A. Silverman, Integral, measure and derivative: a unified approach, Dover Publications, INC. (1977), 209-215. MR 57:6342

[11] M. Su, Measured solenoidal Riemann surfaces and holomorphic dynamics, Journal of Differential Geometry (47) (1997), 170-195. MR 99a:58133

[12] _ Laminations for hyperbolic measures, Preprint, May 2003.

[13] M. Su and D. Sullivan, Laminations for endmorphisms, in preparation, 2003.

[14] D. Sullivan, Bounds, quadratic Differentials, and Renormalization Conjectures, Math. into the Twenty-first Century (Vol. 2), Providence, RI, AMS (1991). MR 93k:58194

[15] _ Linking The Universalities of Milnor-Thurston, Feigenbaum and Ahlfors-Bers: topological methods in modern mathematics, Publish or Perish Inc. (1993). MR 94c:58060

Mathematics Department, Long Island University, Brooklyn Campus, 1 University Plaza, Brooklyn, New York 11201

E-mail address: msu@liu.edu 